\title{
A giant ovarian mass in a 68-year-old female with persistent abdominal pain and elevated serum CA-125 level
}

\author{
Francesk Mulita, Panagiotis Tavlas, Ioannis Maroulis \\ Department of Surgery, General University Hospital of Patras, Patras, Greece
}

\begin{abstract}
Introduction: Ovarian masses are common findings in general gynecology. However giant ovarian mass is a rare finding. In the literature, a few cases of giant ovarian mass have been mentioned sporadically, especially in elderly patients.

Case report: We report a 68-year-old postmenopausal woman with a giant right ovarian mass measuring $38 \times 31 \times 29 \mathrm{~cm}$. She presented to our department with complaints of massive abdominal distention which started gradually 6 months ago. The patient also complained of difficulty in breathing and ambulation. There were no other gastrointestinal, urinary, or gynecological symptoms CA-125 marker was slightly elevated. Because of elevation of serum CA-125 levels, ovarian malignancy was included in differential diagnosis. We performed an ovarian cystectomy without any significant complication. On histopathological examination, the mass was confirmed as benign serous cystadenoma of the right ovary.

Conclusions: Giant ovarian mass is a rare finding in general gynecology. Physicians must maintain heightened awareness and index of suspicion when approaching a woman with pain in any region of the abdomen or pelvis. Further investigation with abdominal and pelvic ultrasonography and magnetic resonance imaging or computed tomography is necessary. Benign lesions can be found even in patients presenting with giant masses and higher CA-125 than normal levels.
\end{abstract}

Key words: cystadenoma, ovarian, mass, serous, CA-125.

\section{Introduction}

Ovarian masses are common findings in general gynecology [1]. Epithelial neoplasms of the ovary account for $60 \%$ of ovarian masses. Ovarian cystadenomas are common benign epithelial neoplasms which have a very good prognosis. Serous and mucinous are the two most frequent types of cystadenomas [2]. These tumors are not usually symptomatic. Abdominal pain and abdominal distension are the two most frequent symptoms and usually appear when these tumors become large [3]. Abdominal and pelvic ultrasonography (US), magnetic resonance imaging (MRI) and computed tomography (CT) are useful tools in making the initial diagnosis of an ovarian mass [4]. In this report, we discuss a case of large serous cystadenoma, which was diagnosed in a 68-year-old female patient.

\section{Case report}

A 68-year-old postmenopausal woman presented to our department with complaints of massive abdom- inal distention which started gradually 6 months ago. The patient also complained of difficulty in breathing and ambulation. There were no other gastrointestinal, urinary, or gynecological symptoms. The woman had a significant past medical history of hypercholesterolemia and her past surgical history included one cesarean section 40 years ago. Her family history is negative for malignant ovarian and breast cancer in first-degree relatives.

On physical examination, the patient's abdomen was grossly distended, hard and tense on palpation. Blood analysis revealed hemoglobin of $11.4 \mathrm{~g} / \mathrm{dl}, 9170$ leukocytes and 235000 platelets. All tumor markers were within normal limits. However CA-125 was slightly elevated $(42.4 \mathrm{U} / \mathrm{ml})$. An abdominal and pelvis magnetic resonance imaging (MRI) was performed. The MRI revealed a huge pelvi-abdominal mass measuring $34 \times 31$ $\times 27 \mathrm{~cm}$, which originated from the right ovary (Fig. 1).

Because of elevation of serum CA-125 levels, ovarian malignancy was included in differential diagnosis. The patient was operated for exploratory laparotomy with removal of right ovarian mass with ovarian cys- 


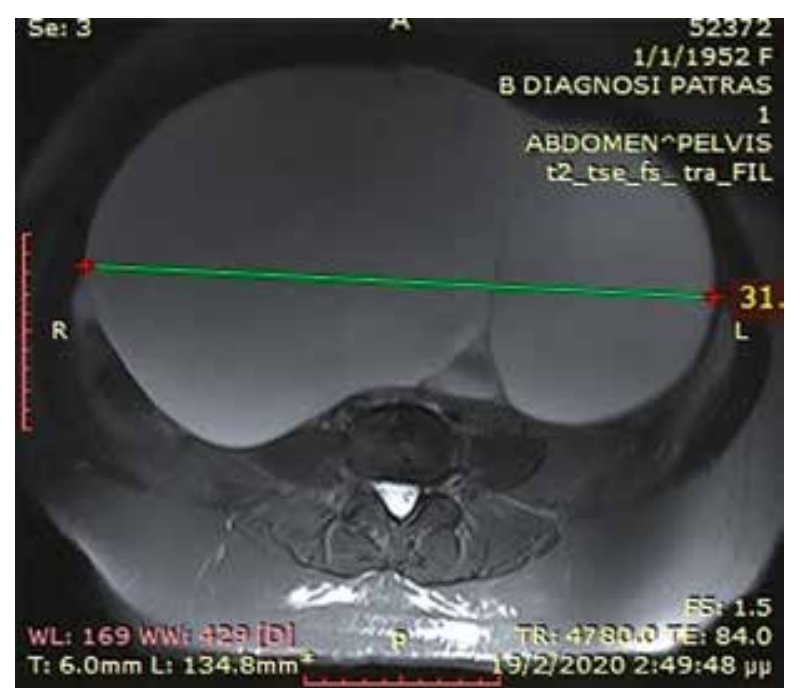

Fig. 1. Abdominal and pelvic MRI revealed a giant mass arising from the right ovary

tectomy (Fig. 2). The patient had no intra-operative or postoperative complications. On day 5 of surgery, patient was discharged home in good condition. Her final histopathology report was suggestive of serous cystadenoma of right ovary.

\section{Discussion}

Ovarian serous cystadenomas, also known as serous cystadenoma, are the most common ovarian neoplasms. They are benign lesions and represent $16 \%$ of all ovarian epithelial neoplasms [5]. Its prevalence peaks between 60-70 years of the human lifespan. Serous ovarian cystadenocarcinomas account for $\sim 25 \%$ of serous tumors [6]. Tumor markers, such as CA-125 can be a useful tool that helps to distinguish between benign and malignant ovarian masses [7]. CA-125 levels of less than $35 \mathrm{U} / \mathrm{mL}$ are now accepted as normal. Elevated levels of CA-125 are more strongly associated with serous, rather than mucinous tumors [8]. It is now widely accepted that the tumor marker CA-125 is a predictive and prognostic factor in CA-125 positive ovarian cancers. Serum CA-125 level is a strong prognostic factor for overall survival and progression free survival in ovarian cancer. There is an inverse relationship between serum CA-125 levels and survival in ovarian cancer. That means that a decreasing level generally indicates a positive response to cancer therapy while an increasing level indicates tumor recurrence and poor survival [9]. However, a few prospective studies indicated the inadequate sensitivity of CA-125 in the setting of ovarian cancer screening in asymptomatic populations [10-12]. Some authors have described elevated levels of these markers in patients with benign tumors [7]. The combi-

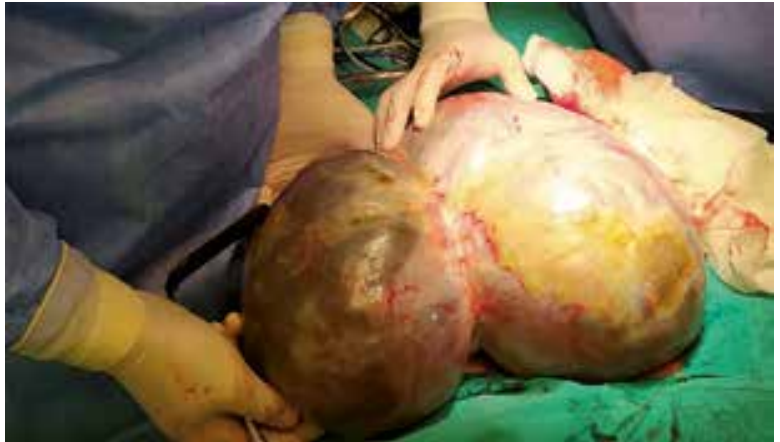

Fig. 2. Completely resection of the ovarian mass that weighed $9 \mathrm{~kg}$ and measured $38 \times 31 \times 29 \mathrm{~cm}$

nation of normal findings at serum CA-125 assay, imaging, and clinical findings exclude the possibility of ovarian cancer [13]. Abdominal and pelvic ultrasonography (US) seems to be useful in making the initial diagnosis of an ovarian mass. Furthermore, magnetic resonance imaging and computed tomography are helpful in confirming the diagnosis of these masses [4].

Surgery is the treatment of choice for serous cystadenomas. Ovarian cystectomy or unilateral salpingo-oophorectomy is commonly considered in huge tumors. Clinical recurrence of serous cystadenomas is not very common and reflects either incomplete resection or a new primary tumor $[2,14]$. Until now, there has been no randomized controlled trial for the laparoscopic management of ovarian cysts $>20 \mathrm{~cm}$, so laparotomy remained the ideal method for the excision of the giant ovarian cysts [15].

Ovarian masses will continue to be a risk for woman, as the majority of patients will not be diagnosed at an early stage. It is suggested that every female patient with pain in any region of the abdomen or pelvis be at least evaluated by imaging techniques.

\section{Conclusions}

Giant ovarian mass is a rare finding in general gynecology. Physicians must maintain heightened awareness and index of suspicion when approaching a woman with pain in any region of the abdomen or pelvis. Further investigation with abdominal and pelvic ultrasonography and magnetic resonance imaging or computed tomography is necessary. Although tumor markers such as CA-125 can be a useful tool to distinguish between benign and malignant ovarian masses, they are not very specific and sensitive for malignancy.

\section{Disclosure}

The authors report no conflict of interest. 


\section{References}

1. Agah J, Jafarzadeh Esfehani R, Kamalimanesh B, et al. Mismanagement of a Huge Ovarian Serous Cystadenoma in a Young Girl; a Case Report. J Midwifery Reproductive Health 2015; 3: 315-317.

2. Limaiem F, Mlika M. Ovarian Cystadenoma. [Updated 2019 Nov 24]. In: StatPearls [Internet]. StatPearls Publishing, Treasure Island (FL) 2020.

3. Ciftci I, Sekmenli T, Ugras S. Ovarian Huge Serous Cystadenoma in Adolescent Girl: A Case Report. Natl J Med Res 2013; 3: 187-189.

4. Mülayim B, Gürakan H, Dagli V, et al. Unaware of a giant serous cyst adenoma: a case report. Arch Gynecol Obstet 2006; 273: 381-383.

5. Seidman JD, Mehrotra A. Benign ovarian serous tumors: a re-evaluation and proposed reclassification of serous "cystadenomas" and "cystadenofibromas”. Gynecol Oncol 2005; 96: 395-401.

6. Ros PR, Mortele KJ. CT and MRI of the abdomen and pelvis, a teaching file. Lippincott Williams \& Wilkins 2006.

7. Katke RD. Giant mucinous cystadenocarcinoma of ovary: A case report and review of literature. J Midlife Health 2016; 7: 41-44.

8. Hogdall EV, Christensen L, Kjaer SK, et al. CA125 expression pattern, prognosis and correlation with serum CA125 in ovarian tumor patients. From The Danish “MALOVA” Ovarian Cancer Study. Gynecol Oncol 2007; 104: 508-515.

9. Gupta D, Lis CG. Role of CA125 in predicting ovarian cancer survival a review of the epidemiological literature. J Ovarian Res 2009; 2: 13.

10. Skates SJ, Menon U, MacDonald N, et al. Calculation of the risk of ovarian cancer from serial CA-125 values for preclinical detection in postmenopausal women. J Clin Oncol 2003; 21: 206s-210s.

11. Nossov V, Amneus M, Su F, et al. The early detection of ovarian cancer: from traditional methods to proteomics. Can we really do better than serum CA-125? Am J Obstet Gynecol 2008; 199: 215-223.

12. Helzlsouer KJ, Bush TL, Alberg AJ, et al. Prospective study of serum CA-125 levels as markers of ovarian cancer. JAMA 1993; 269: 11231126.

13. Jeong YY, Outwater EK, Kang HK. Imaging evaluation of ovarian masses. Radiographics 2000; 20: 1445-1470.

14. Schrecengost A. Ovarian mass - benign or malignant? AORN J 2002; 76: 789-806.

15. Alobaid A, Memon A, Alobaid S, Aldakhil L. Laparoscopic management of huge ovarian cysts. Obstet Gynecol Int 2013; 2013: 380854. 